\title{
VOLUMEN 4 № 2
}

$$
\text { c. } 2
$$

CIENCIA

$\mathrm{E}$

INVESTIGACION

\section{FORESTAL}

DICIEMRRE 1990

INSTITUTO FORESTAL - CHILE

FILIAL CORFC 
DESARROLlO DEL BOSQUE NATIVO - PROBLEMATICA A NIVEL MUNDIAL (Conferencia en el marco del simposio forestal - Mejoramiento del Bosque nativo. Univ. de Talca, Mayo 1990) - Dr. Hans Grosse, Ingeniero Forestal - Instituto Forestal.

\section{INTRODUCCION}

A pocos años del cambio al siguiente milenio, las preocupaciones principales de la humanidad son la escases de recursos, los problemas del medio ambiente y de la población.

En el pasado existió gran abundancia de recursos naturales para cubrir las necesidades de los seres humanos, pero estos cada día son más escasos y estan sometidos a una presion creciente. Diversos organismos nacionales e internacionales han dado a conocer a sus gobiernos y a la opinión pública los antecedentes que demuestran la dimensión del problema.

Un completo diagnóstico fue solicitado en el año 1977 por el entonces presidente del los EE.UU. J. Carter, resultando en 1980 el informe al presidente "Global 2000". En éste se incluyen distintas proyecciones e interrelaciones hasta el año 2000 para los temas referentes a los recursos naturales renovables y no renovables, la energía, el medio ambiente y el ser humano. A pesar de lo dramático que resultó el análisis y pronóstico, los esfuerzos desplegados para mejorar la situacion han sido absolutamente insuficientes durante el decenio de los ochenta. En gran medida esto se debe a la gran complejidad de las situaciones y sus interrelaciones y a la falta de voluntad real de dar solución a los problemas.

Con el objetivo de sugerir un plan de acción que permita resolver los problemas planteados, los autores L. Wicke y J. Hucke diseñan el "Plan Marshall Ecológico", publicado a fines de 1989. El concepto del plan se basa en la idea del programa de recuperación de Europa aplicado después de la Segunda Guerra Mundial. Este consistio en la ayuda material y economica por parte de EE.UU. a los países destruidos por la guerra, recuperando y aumentando posteriormente el capital invertido al normalizarse las economías dentro de Europa Occidental. Los autores proponen que para resolver los graves problemas del medio ambiente, fundamentalmente los relacionados con la reducción de masa boscosa, los cambios climáticos y el ahorro de energía, debe llevarse a cabo una masiva ayuda económica de los países desarrollados. En gran medida los fondos deben ser canalizados hacia los países en los cuales se concentran los problemas, que son principalmente los subdesarrollados, hasta equilibrarse la situacion. El beneficio final también es para los países que realizan el aporte inicial. 


\section{EL ESCENARIO MUNDIAL}

Para poder comprender lo que significa la presion sobre el recurso forestal, resulta indispensable conocer cómo evolucionará el crecimiento poblacional en función del desarrollo de las regiones. dinámica.

Esta información se complementa con el análisis de la existencia forestal y su

Como información base para este capítulo se recurrio al texto del informe "Global $2000 "$.

\section{La población mundial}

Hacia el fin del siglo XX el crecimiento poblacional sigue un ritmo alarmante.

Del ano 1975 al año 2000 el incremento será entre el $46 \%$ y el $55 \%$, lo que significa un aumento desde 4.100 millones a 5.98666 .355 millones de habitantes, respectivamente.

Mientras que los países desarrollados incrementarán su número de habitantes en forma moderada, a una tasa del $0,6 \%$ anual, los países en vías de desarrollo lo harán con una cifra bastante más alta, a un $2,1 \%$ anual. Dentro de estos ultimos destacan las macroregiones Africa, con un 2,9\% y America Latina, con un 2,7\% de incremento anual.

Para America Latina esta tasa significa prácticamente duplicar sus 325 millones de habitantes del ano 1975 en el transcurso de 25 años.

Dentro del contexto mundial, la participación en número de habitantes de los países subdesarrollados muestra un aumento significativo, del $66 \%$ en el año 1950 se estaría llegando a un $79 \%$ en el año 2000.

Serios problemas sociales acompañan al alto crecimiento poblacional. Mientras para las naciones desarrolladas se pueden esperar ingresos anuales percápita, hacia fines de siglo, de US\$8.500, en los países subdesarrollados el ingreso bordeará los US\$600. Se estima que la region sudamericana estaría en mejor pie que otras áreas en vías de desarrollo, pronosticándose un alto aumento del producto social bruto, lo que permitiría optar a un ingreso promedio más alto que el mencionado. 
A pesar de esto, los problemas sociales en las grandes ciudades y en los sectores rurales son de dificil solución. La mayor parte de la población vivirá en los sectores perifericos pobres de las grandes ciudades, debido a las reducidas opciones de trabajo en los sectores rurales.

Paralelo a este fenomeno, la población busca opciones de producción en los bosques del cinturón tropical, aplicando conceptos de utilización de corto plazo, dirigidos a la subsistencia inmediata.

\section{La existencia de bosques}

Según las estimaciones entregadas en el texto Global 2000, aproximadamente en el año 1956 los bosques cubrían el 25\% de la superficie terrestre; el año $1978 \mathrm{el} 20 \%$, pronosticándose para el año 2000 un $17 \%$ y para el año 2020 un 14\% (Cuadrol). Esta última superficię, que tendería a estabilizarse, se compone de áreas en paises desarrollados que actualmente ya presentan una superficie estable, y de sectores de difícil acceso y en consecuencia de baja rentabilidad, en paises subdesarrrollados.

Cuadro 1

EXISTENCIA DE BOSQUES CERRADOS

(Fuente: Global 2000)

\begin{tabular}{|c|c|c|}
\hline Año & $\begin{array}{c}\text { * Bosque cerrado } \\
\text { en miles de } \\
\text { mill. de ha }\end{array}$ & $\begin{array}{c}\text { * Valor relativo } \\
\text { a superf. } \\
\text { terrestre }\end{array}$ \\
\hline 1956 & 3,21 & $25 \%$ \\
1978 & 2,57 & $20 \%$ \\
2000 & $2,10-2,20$ & $17 \%$ \\
2020 & 1,80 & $14 \%$ \\
\hline
\end{tabular}


* Bosque cerrado:

En EE.UU. se define como el bosque cuyo volumen se incrementa anualmente sobre $1,4 \mathrm{~m}^{3} \mathrm{ha}$. En Canadá, como el bosque que en más del $10 \%$ de su superficie presente árboles con un DAP > 10 $\mathrm{cm}$. En el resto del mundo se considera como tal un bosque con una cobertura de copa de sobre el $20 \%$.

** Valores relativos: Sobre base de 13,003 mil mill. ha (19\% superficie artica, sin antártida, Groenlandia y Spitzbergen).

Para el año 1978 se estimó que 1,45 mil millones de hectáreas (aprox. el 56\% de la superficie de bosque cerrado), podian ser consideradas como bosques estables en los paises desarrollados. Si esta superficie se mantuviera constante y la reducción de la superficie de bosques se produjera según el pronostico presentado en el Cuadro 1, es fácil deducir que la eliminación de bosques ocurrirá mayoritariamente en los países subdesamollados. De los 1,12 mil millones de hectáreas de bosque cerrado que había el año 1978, sólo quedarán alrededor de 350 millones de hectáreas en el año 2020. Esto significa una reducción del patrimonio forestal en los paises subdesarrollados de prácticamente un $70 \%$, en términos de superficie, en el trascurso de 42 af́os.

El aumento poblacional y la disminución de la superficie de bosques.

La relación entre el número de habitantes y la superficie de bosque cerrado disponible para estos, es un indicador dinámico que refleja la presion sobre el recurso. Su desventaja es que no especifica los volúmenes disponibles.

En los paises desarrollados, por concepto de un aumento del manejo intensivo del recurso bosque, el rendimiento volumétrico tenderá a subir. Por esta razón, un pequeño aumento poblacional y, en consecuencia,de la presión del ser humano sobre el bosque, estaría equiparado por la mayor oferta disponible de madera por unidad de superficie. Esto significa una disminución de 1,2 a 0,9 hectáreas de bosque cerrado por habitante en 42 afos, lo que en términos productivos no tendría mayor significado (Cuadro 2).

Al contrario, en los países subdesarrollados el manejo de los bosques nativos es muy escaso. Prima la eliminación del recurso por extensas quemas y lo que permanece en pie, corresponde a los volúmenes de menor interes comercial. Esto implica, además de la desaparición de grandes superficies de bosques, una baja en el rendimiento en terminos de volumen y valor del remanente despues de la explotación. A esto se agrega un crecimiento poblacional que en $\mathbf{4 2}$ años prácticamente triplica el número original de habitantes. En consecuencia, es muy probable que de las 0,36 hectáreas de bosque cerrado 
por habitante del año 1978, se llegue a solo 0,04 hectáreas en el año 2020 (Cuadro 2).

Esta situación afectará también a los países desarrollados, a pesar de que éstos mantendrán un patrimonio de bosques estable. Dado que este patrimonio no alcanza para su autoabastecimiento, deben recurrir a la importación de madera desde los países subdesarrollados. Al disminuir el volumen disponible en éstos, aumentando simultáneamente la demanda interna, su capacidad exportadora va a tender a desaparecer.

Cuadro 2

\section{RELACION ENTRE EL NUMERO DE HABITANTES Y LA SUPERFICIE DE BOSQUES CERRADOS EN EL MUNDO}

(Fuente: Gobal 2000)

\begin{tabular}{|c|c|c|c|c|c|c|c|c|c|}
\hline \multirow[b]{2}{*}{ AN̂O } & \multicolumn{3}{|c|}{ PAISES SUBDESARROLADOS } & \multicolumn{3}{|c|}{ PAISES DESARROLLADS } & \multirow[b]{2}{*}{$\begin{array}{l}\text { Ne Habit. } \\
\text { (millones) }\end{array}$} & \multicolumn{2}{|l|}{ TOTAL } \\
\hline & $\begin{array}{l}\text { NYHabit. } \\
\text { (millones) }\end{array}$ & $\begin{array}{l}\text { Sup. Bos. } \\
\text { (mil. mill. } \\
\text { ha) }\end{array}$ & $\begin{array}{l}\text { Sup. bos. } \\
\text { por habit. } \\
\text { (ha) }\end{array}$ & $\begin{array}{l}\text { Ne Habit. } \\
\text { (millones) }\end{array}$ & $\begin{array}{l}\text { Sup. bos. } \\
\text { (mil. mill. } \\
\text { ha) }\end{array}$ & $\begin{array}{l}\text { Sup. bos. } \\
\text { por habit. } \\
\text { (ha) }\end{array}$ & & $\begin{array}{l}\text { Sup. bos. } \\
\text { (mil. mill. } \\
\text { ha) }\end{array}$ & $\begin{array}{l}\text { Sup. bos. } \\
\text { por habit. } \\
\text { (ha) }\end{array}$ \\
\hline 1978 & 3.141 & 1,12 & 0,36 & 1.169 & 1.46 & 1.24 & 4.310 & 2,67 & 0,60 \\
\hline 2000 & 4.863 & 0,70 & 0.14 & 1.333 & 1.45 & 1,00 & 6.296 & 2,16 & 0.34 \\
\hline 2020 & 9.258 & 0,35 & 0,04 & 1.595 & 1.45 & 0,21 & 10.853 & 1,80 & 0.17 \\
\hline
\end{tabular}

Tasa de crecimiento poblacional - paises subdesarrollados - paises desarrolledos

$2,1 \% /$ ah้o
$0,6 \% /$ ah̆o

Sup. bosque : Se considera sólo bosque cerrado

La situación de América Latina requiere una atención especial. Tradicionalmente dotada de las reservas forestales más importantes del mundo en el cinturon tropical, tiende en el corto plazo a ser una region con escasez de bosque. De las 725 millones de hectáreas de bosque del año 1978 quedaría, en el mejor de los casos, solo un 44\% en el año 2020. En el peor caso y lamentablemente pareciera ser el pronóstico más realista, quedaría solo un $19 \%$. Esto significa que después de disponer por cada habitante de casi el doble de superficie de bosques que el mundo desarrollado, con 2,1 hectáreas, se bajaría para el año 2020, a solo 0,13 a 0,29 hectáreas por persona (Cuadro 3). 
Cuadro 3

RELACION ENTRE EL NUMERO DE HABITANTES Y LA SUPERFICIE DE BOSQUES CERRADOS EN AMERICA LATINA

(Fuente: Global 2000)

\begin{tabular}{|c|c|c|c|c|c|}
\hline \multirow[t]{2}{*}{ Ano } & \multirow[t]{2}{*}{$\begin{array}{l}\mathrm{N}^{2} \text { habit. } \\
\text { (millones) }\end{array}$} & \multicolumn{2}{|c|}{$\begin{array}{l}\text { Superficie de bosque cerrado } \\
\text { (mil mill. de ha.) } \\
\text { A tasa de destrucción }\end{array}$} & \multicolumn{2}{|c|}{$\begin{array}{l}\text { Superficie de bosque } \\
\text { por habitantes (ha) } \\
\text { A tasa de destrucción }\end{array}$} \\
\hline & & $2 \%$ & $4 \%$ & $2 \%$ & $4 \%$ \\
\hline 1978 & 352 & \multicolumn{2}{|c|}{0,725} & \multicolumn{2}{|c|}{2,06} \\
\hline 2000 & 632 & 0,469 & 0,306 & 0,74 & 0,48 \\
\hline 2020 & 1.078 & 0,316 & 0,140 & 0,29 & 0,13 \\
\hline
\end{tabular}

Tasa de crecimiento poblacional : $2,7 \%$ / ano

\section{CONSECUENCIAS PARA EL MEDIO AMBIENTE}

Las consecuencias para el medio ambiente, provocadas por la masiva destrucción de bosques, están a la vista de la opinión pública que tenga una mínima sensibilidad para captarla. Reiteradas informaciones a través de los medios de difusión, permiten que las personas más alejadas de estos problemas puedan entender que algo no anda bien. A pesar de que falta mucho por conocer del tema, con el estado del conocimiento actual no debería existir duda de que el planeta se encuentra en estado de alarma y que muchos daños causados por el hombre son ya irreparables.

En el presente capitulo se entregan algunos antecedentes sobre la eliminación de especies, perdidas de suelos y posibilidades de cambios climáticos.

\section{La eliminación de especies}

Durante los últimos decenios ha ido aumentando progresivamente la eliminación de especies.

Según Lovejoy (1980) aún cuando la destrucción de bosques sea baja, se debe pensar que hasta fines de siglo se eliminaría el $15 \%$ de las especies existentes. Si la destrucción es alta alcanzaría al 20\%. Esto significa que se eliminarán entre 500.000 y 6.000 .000 de especies, debiéndose el amplio rango al desconocimiento del número total 
de especies existentes. En primer lugar de la desaparicion figuran los insectos, seguidos por las plantas. Para los últimos veinte años del presente siglo esto significa una eliminación de 68 a 822 especies por día. La explicación para llegar a números tan elevados es la gran cantidad de especies existentes en el cinturón de bosques tropicales, que se estima que podrían llegar al $80 \%$ de la existencia mundial.

Con este proceder se imposibilita a la humanidad conocer y aprovechar las especies vivas, que se extinguen y pasan a ser un recurso definitivamente no renovable.

\section{El problema de los suelos y la erosión}

Es un fenomeno ampliamente conocido que gran parte de los ríos que evacuan aguas de las zonas tropicales y subtropicales con la más mínima lluvia cambian su transparencia a un color café barroso. Esto es el típico síntoma de que sus cuencas están sufriendo fuerte erosión por la eliminación de la capa vegetal por el hombre.

En busca de terrenos para la agricultura, grandes roces a fuego ponen al descubierto los suelos, los cuales, en el sector tropical, mayoritariamente no sirven para cultivos en el largo plazo. Interrumpido el ciclo de nutrientes y agregando el lavado de los suelos, rápidamente estos empobrecen y se transforman en áreas cubiertas de cárcavas endurecidas por el carácter laterítico de gran parte de ellos. Al haberse perdido la masa vegetal capaz de absorber las aguas lluvias se producen fuertes caudales en el desagüe y se producen grandes inundaciones. A su vez, al perderse el efecto acumulador de los bosques, no existen reservas de agua, secándose vertientes y arroyos en epocas de sequía.

Las pérdidas de suelo que se pueden producir por tala rasa sin reponer el bosque, para un ejemplo de la Costa de Marfil, son según Wicke y Hucke (1989) de 90 ton/ha al año, contra 0,03 ton/ha al año para sectores intactos. Al llevar el proceso erosivo a un plazo de 10 ańos, la pérdida acumulada estaría representada por 900 ton/ha.

Un estudio reciente realizado por Alvarez (1989), entrega datos sobre los efectos de la erosión después de tala rasa en la zona costera de la VIII Región en Chile. El material perdido en terrenos que permanecen sin vegetación supera ampliamente los márgenes considerados como tolerables, de 2,5 - 4,0 ton/ha al año (Cuadro 4). El problema de la erosión en Chile, está dimensionado por los 19 millones de hectáreas rozadas con fuego, donde han quedado profundas huellas en el paisaje (Trivelli, 1970). 
El embancamiento de ríos que eran conocidos por su navegabilidad, como el Maule y el Bio - Bio, entre otros, ejemplos que ilustran parte de la pérdida económica que significa el proceso erosivo para el país (Elizalde, 1970).

Cuadro 4.

PERDIDA DE SUELO EN FUNCION DE LA PENDIENTE (en ton/ha) (Alvarez, 1989).

\begin{tabular}{|c|c|c|c|}
\hline $\begin{array}{c}\text { Longitud } \\
\text { Pendiente } \\
(\mathrm{m})\end{array}$ & $20 \%$ & $40 \%$ & $60 \%$ \\
\hline 100 & $26 \cdot 35$ & $69 \cdot 92$ & $122 \cdot 163$ \\
200 & $40 \cdot 53$ & $105 \cdot 140$ & $185 \cdot 246$ \\
400 & $60 \cdot 80$ & $159 \cdot 212$ & $280 \cdot 373$ \\
800 & $126 \cdot 168$ & $241 \cdot 321$ & $425 \cdot 566$ \\
\hline
\end{tabular}

\section{Efectos climáticos}

Uno de los fenomenos relacionados con la pérdida de bosques más comentado en el efecto de invernadero. A continuación se sintetizan algunos aspectos analizados extensamente por Burschel y Weber (1988).

La concentración de $\mathrm{CO}_{2}$ en la atmosfera históricamente era de $270 \mathrm{ppm}$, aumentando en un $20-25 \%$, para llegar a 350 ppm en $50-60$ años. Se estima un incremento anual de 1,3 a $1,5 \mathrm{ppm}$.

La explicación para este aumento se debe al quiebre del equilibrio en el intercambio de $\mathrm{CO}_{2}$ entre la atmósfera, biosfera y los mares. Grandes cantidades de $\mathrm{CO}_{2}$ son liberadas por las quemas y eliminación de biomasa forestal, además de las emisiones provenientes del sector industrial.

$\mathrm{Al}$ acumularse una mayor cantidad de $\mathrm{CO}_{2}$ en la atmosfera, además de otros compuestos gaseosos, aumenta el efecto natural de invernadero. Sin éste la temperatura en el planeta sería de $-18^{\circ} \mathrm{C}$. Se estima que las temperaturas aumentarán en el transcurso del próximo siglo entre 1,5 y $4,5^{\circ} \mathrm{C}$, suponiéndose un cambio más grande hacia los polos.

Las consecuencias para las economías de los países serían dramáticas, si se considera que las zonas áridas y con climas mediterráneos se desplazarían hacia los polos de cada hemisferio. 
Una alternativa para detener este proceso radica en la mantencion de los bosques actuales y la creación de nueva biomasa forestal capaz de fijar $\mathrm{CO}_{2}$. Esto implica revertir el proceso que ocurre actualmente.

\section{EL PLAN MARSHALL ECOLOGICO}

La situación de destrucción de las grandes reservas de bosques que se concentran en los países subdesarrollados sigue una tendencia alarmante. Solo medidas drásticas y eficientes podrán detener este proceso. Los autores Wicke y Hucke (1989) afirman que aun existen esperanzas para resolver estos problemas.

Sugieren un programa que contenga el espíritu del Plan Marshall aplicado después de la Segunda Guerra Mundial, pero en esta oportunidad poniendo enfasis en la reconstrucción de los bosques. Por este motivo su nombre "El Plan Marshall Ecologico". Para su realización deberán considerarse distintas actividades que se desglosan en los puntos siguientes.

Generación de la base para lograr mayor eficiencia en la protección del recurso.

Para la realización del programa se consideran aportes por US\$2.000 millones para el primer año aumentando en US\$ 1.000 millones anualmente, hasta llegar a los US\$ 6.000 millones.

Unos de los problemas de arrastre más grave para las naciones subdesarrolladas es su deuda externa. En este sentido cabe buscar soluciones como cambiar una mayor protección del medio ambiente por parte de la deuda. Otra medida podrían ser aportes directos.

Con estos aportes de capital se crearían las condiciones básicas para revertir el proceso destructivo. Los países que integrarían el programa lo harian en términos voluntarios; una vez integrados en el grupo deberán ceñirse a las reglas del juego, siendo consecuentes con los principios planteados. Por ejemplo, si en un país determinado por un lado se toman medidas adecuadas y por el otro lado se continua con la destrucción, el aporte en dinero podría transformarse en un crédito con altas tasas de interés de mercado, como castigo.

Para enfrentar el programa deberán integrarse grupos técnicos que operen en defensa de la naturaleza, independientes de los gobiernos. A estos grupos se deben agregar 
especialistas de distintas disciplinas, ya que no sollo deberán atacarse los problemas netamente forestales, sino que también otros aspectos que también inciden en la presión sobre el recurso. Sólo un análisis y enfrentamiento del problema en su total magnitud, incluyendo el apoyo técnico de los más calificados especialistas de todo el mundo, tiene posibilidades de éxito.

Programas de ocupación de mano de obra en las zonas rurales

Con el objetivo de disponer reservas de leffa y madera, incluyendo esquemas que involucran actividades agrícolas y forestales, deben realizarse amplios programas de reforestacion. En estos se ocuparfa gran cantidad de mano de obra joven, cesante, de las áreas rurales. En estos programas también deberá recurrirse a especies exóticas que puedan cumplir en un plazo menor con la demanda existente. De este modo se baja la presión sobre el bosque nativo remanente.

También, con el objeto de proveer energía y reducir la presión sobre los bosques naturales, se deberán poner en práctica sistemas integrados de producción, los cuales incluyan una componente forestal en las actividades agrícolas y ganaderas. Ejemplo de esto son las cortinas cortavientos, los bosquetes de abrigo para el ganado y otras muchas combinaciones.

\section{Mejorar condiciones de marco}

Se deberán mejorar las condiciones que enmarcan y presionan los recursos forestales nativos. Dentro de este cuadro se mencionarán sin entrar a fondo los temas principales:

- Se deberán reforzar y mejorar las instituciones de importancia para el sector forestal, ya sean de coordinacion, control, forestacion, industrias o investigación.

- Otro tema de importancia es la reducción de la pobreza en las zonas rurales, para evitar de este modo la migración a las ciudades. En este tema caben para algunos países consideraciones sobre reforma agraria, capacitación y coordinación para el trabajo en comunidades agrícolas.

- Para contrarestar la presión ejercida sobre el recurso por el crecimiento poblacional desmesurado, se propone incorporar masivos programas para controlar la natalidad. Complementaria a esta medida se sugiere buscar posibilidades de pago de jubilaciones que descarten que ciertos grupos familiares tengan hijos como un seguro para la vejez. 


\section{Protección especial de la selva lluviosa de Brasil}

La selva tropical del Brasil, con sus 357 millones de hectáreas, es la unidad forestal más importante de Sudamérica. Con las regiones selváticas de los países limitrofes prácticamente se duplica esta cifra. Dentro de este patrimonio forestal, Brasil presenta ciertos problemas especiales como son :

- Cesantía rural por cambios de cultivos intensivos a extensivos (caña de azúcar para la obtencion de alcohol metílico).

- Necesidades energéticas. Grandes represas cubrirían extensas superficies de bosques.

- Masivo uso de lefna en la minería.

- Plan de construcción de una carretera que una Perú con Brasil, que se teme incremente la destrucción de bosques.

- Cambio de bosques a superficies de pastoreo.

- Críticas al uso de energía atómica como alternativa al uso de la biomasa.

Frente a esta situación, Brasil y algunos países limítrofes requieren urgentemente de una eficiente ayuda.

Un alto nivel de deuda externa complica las soluciones en el corto plazo. Aún cuando Brasil hiciera todo el esfuerzo posible para ahorrar energía, aprovechar en mayor medida la energía solar y ampliar su capacidad instalada productora de energía atómica, requiere de la colaboracion del mundo desarrollado para salvar su patrimonio forestal.

Los problemas sociales y forestales del mundo subdesarrollado no soblo afectan a estos países, síno que también a los desarrollados. Razón por la cual se justifica ampliamente un programa de ayuda que en definitiva beneficiaría a todos.

\section{Los fondos propuestos para el programa}

Los aportes propuestos por Wicke y Hucke (1989) completan, para un período de 20 años, 800 mil millones de US\$. Durante los primeros dos años esto significa aportes anuales por US\$ 10 mil millones que aumentarían hasta llegar a US\$ 50 mil millones (Cuadro 5). 


\section{APUNTES}

Cuadro 5

APORTES PARA EL PLAN MARSHALL ECOLOGICO

(en miles de millones de US\$ por año)

\begin{tabular}{|l|c|c|c|c|c|c|}
\hline ITEM & $1990 / 1$ & $1992 / 3$ & $1994 / 5$ & $1996 / 7$ & $1998-2009$ & $1990-2009$ \\
\hline $\begin{array}{l}\text { Base para } \\
\text { mayor } \\
\text { eficiencia }\end{array}$ & 2 & 3 & 4 & 5 & 6 & 100 \\
\hline $\begin{array}{l}\text { Prog. ocup. } \\
\text { mano obra }\end{array}$ & 2 & 4 & 8 & 10 & 10 & 168 \\
\hline $\begin{array}{l}\text { Mej. cond. } \\
\text { Marco }\end{array}$ & 4 & 9 & 14 & 20 & 26 & 406 \\
\hline $\begin{array}{l}\text { Prot. esp. } \\
\text { selva Trop. }\end{array}$ & 2 & 4 & 4 & 5 & 8 & 126 \\
\hline TOTAL & 10 & 20 & 30 & 40 & 50 & 800 \\
\hline
\end{tabular}

Para obtener estos fondos se propone optar por un cobro según emisión de $\mathrm{CO}_{2}$ en el consumo energético.

Del dinero recaudado $2 / 3$ se dedicaría a resolver problemas ecologicos internos y $1 / 3$ para el Plan Marshall Ecologico.

De llevarse a cabo este programa se estima que a partir del año 2, el aporte por grupo familiar en los países desarrollados alcansaría a US\$19,4 mensuales y a partir del año 10 a US\$ 55,6 .

Si bien estos sacrificios aparecen como bastante altos, los beneficios a los que puede optar toda la humanidad superan con creces la inversion.

\section{La aplicación del Plan Marshall Ecológico en política internacional}

Los fondos para el programa provendrían de los países afiliados al OECD (Organización para la Colaboración Económica y el Desarrollo). En una acción conjunta de UNDP (United Nations Development Programme) y de IDA (International Develop- 
ment Association) se debe generar la seccion "Fondos para el Plan Marshall Ecológico" en el Banco Mundial.

Desde aqui se organizaría la entrega de fondos, las devoluciones de estos y las sanciones. Como una base se considerarían los tratados internacionales existentes, coordinando las actividades entre organizaciones como FAO, UNDP, UNEP (Programa para el medio ambiente de las Naciones Unidas) e ITTO (Programa para la organización de los trópicos).

El programa base generado por TWAP (Plan de Acción de los Trópicos), que se encuentra en vigencia, debería ser completado y mejorado. La acción se coordinaría en un nivel macro con el objetivo de detectar y llevar los recursos a las áreas prioritarias.

La coordinación del programa es una tarea difícil debido principalmente a:

- Coordinación de muchas intituciones y disciplinas.

- Los gobiernos de los países donantes deben convencerse que es un sacrificio necesario.

- Los países afiliados a OECD deben ponerse de acuerdo.

\section{CONCLUSIONES Y RECOMENDACIONES}

Los antecedentes entregados permiten concluir y recomendar lo siguiente :

- En el planeta se está produciendo una serie de efectos catastroficos originados por las alteraciones que el hombre ha provocado en la naturaleza. Estos se reflejan en procesos erosivos por las perdidas de bosques, inundaciones, sequías y hambrunas en sectores puntuales, y en macroefectos que inciden sobre las interrelaciones globales del planeta, como es el efecto de invernadero.

- El pronóstico es poco alentador, ya que se vislumbra un incremento de las catástrofes naturales, acompanadas por un alto crecimiento poblacional en los países subdesarrollados, que implica un aumento de la presión sobre el recurso bosque. A su vez, el número de personas en extrema pobreza sigue creciendo. Esta situación atenta tanto contra la vida del hombre y su dignidad, como contra toda la vida del planeta. 
-Frente a esta dramática situación existen esperanzas para resolver los problemas. Como premisa básica debe existir la voluntad para hacerlo, reduciendo los egoismos personales y nacionales.

- La sugerencia de realizar un Plan Marshall Ecologico, que incorpore toda las áreas de incidencia en los problemas, buscando resolverlos en forma coordinada y asumiendo sacrificios inmediatos con inversiones dirigidas hacia los puntos neurálgicos, aparece como una posibilidad para revertir la tendencia actual. Esto significa una participación conjunta de los países desarrollados y subdesarrollados.

- Dentro de las medidas previstas, se consideran parques nacionales, reservas y otras áreas de protección que cubrirían una superficie mínima. Mayoritariamente la superficie con bosques nativos del mundo va a tener que ceder a la presion originada por las necesidades energéticas y de madera de los seres humanos. Esto no significa necesariamente la destrucción de éstos, sino que su incorporación como áreas productivas a través del manejo racional del recurso.

En algunos sectores van a ser reemplazados por plantaciones de rápido crecimiento. Sobre todo en sectores ya destruidos y mal utilizados por faenas agrícolas y de pastoreo. En otros de menor alteración se podrá optar por el manejo de las especies autóctonas, tomando en cuenta el beneficio que significa minimizar la alteración de los ecosistemas.

El bosque y los sistemas dependientes de éste, incluyendo la componente humana, solo tienen oportunidad de sobrevivir, si se encuentran soluciones globales a los problemas. Aún se está a tiempo para actuar, pero el tiempo se está terminando. Esto significa que acciones eficientes deben ser tomadas en todos los países del mundo en el corto plazo.

\section{REFERENCIAS}

ALVAREZ F. (1989) : Evaluación de erosión producida por actividades forestales productivas. 2da. etapa. CONAF (UMDEF), Progr. Manejo de Cuencas VIII Region. 30 pp.

BURSCHEL P. y WEBER M. (1988) : Der Treibhauseffekt. Bedrohung und Aufgabe für die Forstwirtschaft. AFZ. Nr. 37. BLV Verlagsgesellschaft. D-8-München-40. Sonderdruck.

COUNCIL ON ENVIRONMENTAL QUALITY (1980): GLOBAL 2000. Der Bericht an den Präsidenten. Greno GMBH. D-6053 Obertshausen. 1508 pp. 
ELIZALDE R. (1970) : La sobrevivencia de Chile. Minist. de Agricultura. SAG. STGO: CHILE. 492 pp.

LOVEJOY T. (1980) : Eine Prognose der Artenverringerung. (En GLOBAL 2000. Greno Gmbh. D-6053. Obertshausen.) (pág. 690-697).

TRIVELLI H. (1970) : Prólogo en "La Sobrevivencia de Chile". Minist. de Agricultura. SAG. STGO: CHILE. XIX - XXVII.

WICKE L. y HUCKE J. (1989) : Der ökologische Marshallplan. Ullstein Gmbh. Frankfurt / Main. Berlin. 336 pp. 